\title{
Clinician advice, an interactive computer program, and motivational counselling during routine medical visits increased reported smoking abstinence among teens
}

Hollis JF, Polen MR, Whitlock EP, et al. Teen reach: outcomes from a randomized, controlled trial of a tobacco reduction program for teens seen in primary medical care. Pediatrics 2005;115:981-9.

Does an intervention comprising brief clinician advice during routine medical visits, an interactive computer program, and brief motivational counselling reduce smoking in adolescent smokers and non-smokers over the long term?

\section{METHODS}

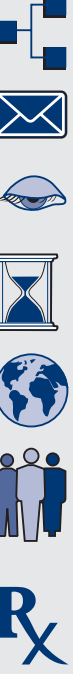

Design: randomised controlled trial (Teen Reach programme).

Allocation: \{concealed\}.*

Blinding: blinded \{data collectors and outcome assessors\}.*

Follow up period: 1 and 2 years.

Setting: 7 large paediatric and family practice departments in a group practice health maintenance organisation in Portland, Oregon and Vancouver, Washington, USA.

20. Participants: 2526 adolescents $14-17$ years of age $159 \%$ girls, $78 \%$ white) who were willing to stay after their clinician visit for about 15 minutes and did not intend to leave the geographic area in the next year.

$\mathbf{R}_{\mathbf{X}}$

Intervention: 1254 adolescents were allocated to the Teen Reach intervention, which comprised (1) a written prompt for primary care clinicians to encourage teens to quit smoking or to not start (30-60 sec advice message); (2) a 10-12 minute session on the Pathways to Change (PTC) interactive computer program that assessed the stage of readiness to start (or quit) smoking and then provided individualised advice; (3) $3-5$ minutes of motivational counselling by trained health counsellors; and (4) 2 individual booster sessions with the PTC program and the health counsellor (during subsequent visits or by telephone) over the subsequent 11 months. 1272 adolescents were allocated to a diet intervention, which served as an attention control and included 3-5 minutes of motivational counselling by health counsellors to promote increased consumption of fruit and vegetables.

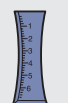

Outcomes: self reported smoking abstinence in the 30 days before 1 and 2 year assessments.

Patient follow up: $94 \%$ at 1 year and $88 \%$ at 2 years. *Information provided by author.

\section{MAIN RESULTS}

Adolescents who received the Teen Reach intervention had higher smoking abstinence rates than those who received the diet intervention at both 1 and 2 years (table). Similar results were found for the subgroup of adolescents who reported smoking $\geqslant 1$ cigarette in the previous 30 days at baseline (ie, experimenters, smokers, and recent quitters). Subgroup analysis of adolescents who did not smoke at baseline showed that those in the Teen Reach group had reduced onset of smoking at 1 year but not 2 years.

For correspondence: Dr J F Hollis, Center for Health Research, Kaiser Permanente, Portland, OR, USA. jack.hollis@kpchr.org

Source of funding: National Cancer Institute.

\section{CONCLUSIONS}

A smoking cessation intervention comprising brief clinician advice during routine medical visits, an interactive computer program, and brief motivational counselling increased reported smoking abstinence among adolescents at 2 years.

A modified version of this abstract appears in Evidence-Based Medicine.

Teen Reach smoking cessation intervention $v$ diet intervention (control) for smoking abstinence in adolescents*

\begin{tabular}{lllll}
\hline Follow up & Teen Reach & Control & RBI $(95 \%$ Cl) & NNT (CI) \\
\hline 1 year & $77 \%$ & $73 \%$ & $6.1 \%(2$ to 10$)$ & $23(14$ to 67$)$ \\
2 years & $73 \%$ & $69 \%$ & $6.2 \%(1$ to 11$)$ & $24(14$ to 158$)$ \\
\hline
\end{tabular}

*Abbreviations defined in glossary; RBI, NNT, and Cl calculated from control event rate and odds ratio (CI) in article. Based on $n=2524$ with multiple imputation for missing values.

\section{Commentary}

Tobacco is a leading cause of death worldwide. Interventions to prevent smoking uptake are urgently needed. The study by Hollis et $a l$ is an important addition to the evidence. Short follow up $(<6 \mathrm{mo})$ is a limitation of smoking studies, but this study followed up adolescents for 2 years. Study participants were primarily Caucasian, college bound adolescents. More than $50 \%$ of smokers at baseline reported they were trying to stop or had stopped smoking in the past 6 months; less motivated adolescents may prove more resistant to this intervention.

The intervention was tailored to individuals' readiness to change, although recent reviews reveal little difference in the effectiveness of programmes that are based on the Stages of Change model and those that are not. ${ }^{12}$ More importantly, data on smoking status was self reported. Hollis et al justified the use of self report, but a recent study found that $20 \%$ of participants who reported smoking cessation had cotinine levels consistent with smoking; ${ }^{3}$ thus, reliance on self report alone may overestimate effectiveness.

The study was pragmatic rather than clinical and therefore is more likely to be applicable to primary care and community based settings. The positive effect and the brevity of the intervention contribute to its feasibility, and brief provider advice to stop smoking overlaps with current recommended practice.

\section{Dawn Kingston, RN, MSc McMaster University Hamilton, Ontario, Canada}

1 Adams J, White $M$. Why don't stage-based activity promotion interventions work? Health Educ Res 2005;20:237-43.

2 Riemsma RP, Pattenden J, Bridle C, et al. Systematic review of the effectiveness of stage based interventions to promote smoking cessation. BMJ 2003;326:1175-7.

3 Rodgers A, Corbett T, Bramley D, et al. Do u smoke after txt? Results of a randomised trial of smoking cessation using mobile phone text messaging. Tob Control 2005; 14:255-61. 